\title{
Prediction of 24-Hour Urinary Sodium Excretion Using a Single Spot Urine Samples in Moroccan Population
}

\author{
Mohamed Idrissi ${ }^{1,4, *}$, Naima Saeid ${ }^{1}$, Anass Rami ${ }^{\text {, }}$, Mohammed El Mzibri ${ }^{\text {I, }}$ Arthur Assako ${ }^{\text {, }}$, Koussou jahdiel ${ }^{3}$, \\ Asmaa El Hamdouchi ${ }^{~}$, Naima Safsaf ${ }^{1}$, Latifa Belakhel ${ }^{2}$, Hasnae Benkirane ${ }^{l}$, Khalid El Kari ${ }^{1}$, Abdallah Bagri ${ }^{4}$ \\ and Hassan Aguenaou ${ }^{1}$ \\ ${ }^{1}$ Joint Research Unit in Nutrition; Health and Environment, RDC- Nutrition AFRA/IAEA, Laboratory Biology \& Health Ibn \\ Tofaïl University-CNESTEN). \\ ${ }^{2}$ Ministry of Health, Rabat, Morocco \\ ${ }^{3}$ School of nutrition,Food sciences and Technologies,Faculty of agronomics Sciences, Abomey-Calavi,Republic of Benin \\ ${ }^{4}$ Laboratory of Biochemistry and Neurosciences, Integrative and Computational Neuroscience unit, Faculty of Sciences and \\ Technology, Hassan First University, B.P: 577, Settat 26000, Morocco
}

\begin{abstract}
Background: Excessive sodium intake is linked to high blood pressure. Estimating sodium intake is difficult. The 24-h urine collection is currently the recommended method for estimating intake but cumbersome for large population studies. Predictive model to estimate sodium intake based on single spot urine were developed, but showed inconsistency when used in extern populations. This study aims to develop a specific model for estimating sodium excretion over 24 hours for the Moroccan population. Methods: 371 participants in the urinary validation sub-study of the STEP-wise survey-Morocco 2017-2018 provided a valid 24-hour urine collection and spot urine specimens. Participant were randomly assigned to the training $(n=183)$ and the validation data set $(n=188)$. Results: A prediction model for 24 -hour sodium excretion was developed. Adjusted $\mathrm{R}^{2}$ was 0.258 . In the validation data set, correlation was 0.431 [ $95 \% \mathrm{Cl} ; 0.258-0.580$ ], and the adjusted $\mathrm{R}^{2}$ was 0.190 . The Bland-Altman plot showed a nonsignificant small mean bias of $-18 \mathrm{mg}(95 \% \mathrm{Cl},-213$ to 177$)$ in predicting $24-\mathrm{h}$ urinary sodium excretion at the group level. At the individual level, limits of agreement were wide. Conclusion: This new model developed from a single spot urine could be used to predict the average 24-h sodium excretion of Moroccan adults.
\end{abstract}

\section{Introduction}

Excessive sodium (salt) intake is associated with high blood pressure level and a high risk of cardiovascular events [1-4]. Currently, sodium intake in individuals around the world exceeds their physiological needs (i.e. $200-500 \mathrm{mg} /$ day) [5]. Moreover, estimates of this mean in adults have been found to be equal to $3.95 \mathrm{~g}$ per day [6], almost double the World Health Organization (WHO) recommendations of no more than $2 \mathrm{~g}$ /day of sodium intake (equivalent to $5 \mathrm{~g}$ per day of salt) [7]. Salt reduction is one of the top "best-buy" strategies. WHO sets a global target of reducing the salt consumption of the population by 2025 as part of its fight against non-communicable diseases (NCDs) (WHO, 2020) [8], each country having the possibility of setting its voluntary target. Morocco's strategy is a commitment to reduce the average salt consumption in the population by $10 \%$ by 2029 [9].

The main challenge in implementing the strategies and demonstrating their effectiveness is the use of accurate methods to assess the usual sodium intake of the population and to monitor its changes over time.

Although 24-h urine collection is the most accurate method for assessing dietary sodium intake, it is cumbersome and resource-intensive for large population 
studies [10], especially in the middle- and low-income countries (MLIC) [11].

Thus, there is an urgent need to find more alternative methods to 24-hour urine collection as the WHO deadline approaches [12]. Equations to predict 24-hour urinary sodium excretion (24HUNa) [13-16] have been developed based on spot urinary sodium and creatinine concentrations as well as basic characteristics of individuals such as age, height and weight and/or body mass index (BMI). Recently, numerous studies have examined the validity of previously published predictive equations for estimating $24 \mathrm{HUNa}$ across populations. Investigators found poor agreement between predicted and measured 24HUNa, suggesting the inadequacy of these predictive equations for use in a population different from that in which they were initially developed [17-21]. Thus, it is recommended to develop new population-specific equations. We aim here to develop and validate a Moroccan specific predictive equation for $24 \mathrm{HUNa}$.

\section{Methods}

\subsection{Study design and population}

Data were based on the urinary validation sub-study of the STEP-wise survey-Morocco 2017-2018. Briefly, the STEP-wise survey-Morocco 2017-2018 was a nationwide cross-sectional study that aimed to assess the prevalence of the risk factors of NCDs in Moroccan adults. A total of 6,100 adults were selected to participate in the STEP-wise survey-Morocco 20172018 using the standard WHO STEPS sampling design which is a robust probability-sampling process that allows for the selection of representative data with three sampling stages (cluster, household, and individual).

Data were collected in three steeps: 1) clinical interview including self-reported information on basic characteristics and health behavioral risk factors of participants, 2) blood pressure (BP) and anthropometric measurements, and 3) biochemical analysis (blood and urine). Details on the STEP-wise survey-Morocco 2017 2018 were described elsewhere [22]. A total of 371 individuals (139 Men and 253 women) aged 18 years and over who reside in the rural and urban areas of Rabat-Salé-Kenitra region (the North West region of Morocco) were included in the urinary validation substudy using the same sampling design of the STEP-wise survey-Morocco 2017-2018[22]. Participants were asked to provide a 24-hour urine collection and a spot urine sample. Individuals declared to have kidney diseases or engaged in a salt-free diet, and women in menstruation period were instructed not to collect urine and were excluded from this study. The field work was conducted during March-June 2017. This study was approved by the Biomedical Research Ethics
Committee at the Faculty of Medicine and Pharmacy of Rabat. All participants have signed informed consent.

\subsection{Measurements}

\subsubsection{Urine collection}

Detailed oral and written instructions for 24-hour urine collections and spot urine specimens were presented to individuals who were eligible and willing to participate in the study. The 24-h urine samples were collected according to the WHO/PAHO standard protocol [23]. Participants were asked to discard the first void collected in the morning and keep collecting all urine voided during the rest of the 24-hour period, including the first void of the second day. The time of the start and the finish of the 24-hour collection was recorded by each participant. Participants were instructed to store the urine samples in a dark and cold place. Once finished, the 24-h urine collections were transported in a thermoelectric coolers to the laboratory.

The total volume of the 24-h urine collection was recorded. After shaking, 5-ml aliquots were prepared and immediately frozen at $-21^{\circ}$ for analysis at the laboratories of "Joint Research Unit in Nutrition and Food" Rabat. The evening spot urine specimens were collected one day before the 24-h urine collection as a fraction of a void collected during the evening period between 7 p.m. and 10 p.m. The spot urine specimens were treated using the same methods for the 24-hour urine samples. The completeness of the 24-hour urine collection in the present study was defined as: (1) total urine volume $400 \mathrm{ml} \leq \mathrm{V} \leq 3600 \mathrm{ml}$; (2) collection period $22 \mathrm{~h} \leq$ time $\leq 26 \mathrm{~h}$ (3) participant reported losing more than few urine drops or missing one void.

This approach was used in previous references largescale international epidemiological studies such as INTERALT, INTERMAP and NHANES. Sodium and potassium concentrations were analyzed using the Inductively Coupled Plasma Mass Spectrometry (ICPMS; Thermo Scientific XSERIES2). An international reference material, Seronorm TM Trace Elements Urine, was used to control and validate the measurements. The creatinine excretion was analyzed using the Jaffe method using the Cobas C311 (Roche diagnostic, Meylan-France). The sodium and creatinine concentrations in the 24-h urine collection were adjusted by the total volume of the collection. The coefficients of variation for sodium, potassium and, creatinine were all less than 3\%, indicating a low analytical imprecision.

\subsubsection{Weight, Height and Blood Pressure}

Physical measurements included weight, height, and BP were recorded by a health professional according to the WHO protocol [24] (page 182-184). Height was measured to the nearest $0.5 \mathrm{~cm}$ and weight was measured to the nearest $0.1 \mathrm{~kg}$. Body mass index (BMI) was estimated as weight in kilograms divided by height 
in meter squared $\left(\mathrm{kg} / \mathrm{m}^{2}\right)$. Three brachial BP measurements were recorded using a calibrated digital sphygmomanometer (Digital Automatic Blood Pressure Monitor DABPM Spengler ES 60). Three brachial systolic blood pressure (SBP) and diastolic blood pressure (DBP) records were averaged to determine the mean BP values. All participants completed 3 measurements. Blood pressure was measured in participants in sitting position and appropriate cuff sizes was used. Before BP measurements, participants were rested for at least $5 \mathrm{~min}$. Detailed procedures and equipment's were described in the STEPS manual published by the WHO [25].

\subsection{Statistical analyses}

Data was partitioned randomly (stratified by sex and age) into training $(n=183)$ and validation $(n=185)$ data sets.

\subsubsection{Developing a Moroccan prediction model}

We developed a regression model to predict 24-hour urinary sodium excretion using individuals from the training data set. Similarly to the INTERSALT equation [13], our model was developed using one-step regression, which is an approach expected to reduce the amount of measurement error compared to the two-steps approach used to develop Kawasaki, Mage and Tanaka equations [14-16]. The model was developed using the measured 24-h sodium excretion as the outcome variable. Sodium and creatinine concentrations in the spot urine, age, and BMI, were used as predictors. No interaction was found between different predictors. The inclusion of each parameters as a predictor in the model was based on their known influence on sodium excretion and their significant contribution to the model. Model fit was assessed using adjusted $\mathrm{R}^{2}$.

\subsubsection{Validation of the prediction model}

Regression coefficients obtained from the training data set were used to estimate the 24-h sodium excretion in the validation data set. The measured and predicted $24-$ hour sodium excretion were presented as the mean and standard deviation (SD). Pearson correlation was calculated to assess the association between measured and predicted 24-hour urinary sodium excretion using the newly developed model, and the bias (predictedmeasured) was calculated using paired t-test. The distribution of measured against the predicted 24-hour urinary sodium excretion with the regression line $(95 \% \mathrm{CI})$ and the line of equality (identity line) was visualized using the scatter plot.

The Bland Altman plot [26] [27] was used to assess agreement between the measured and predicted 24-hour sodium excretion. The degree of mean bias was calculated with the $95 \%$ limits of agreement. The $95 \%$ limits of agreement, calculated as $\pm 1.96 \times$ standard deviation of the mean differences should include $95 \%$ of individual bias. The Bland-Altman plot was drawn by plotting the individual bias (pred 24hUNa minus meas$24 \mathrm{hUNa}$ ) on the $\mathrm{Y}$ axis against the individual means [(pred 24hUNa plus meas $24 \mathrm{hUNa} / 2$ ] on the $\mathrm{X}$ axis. A sensitivity analysis was performed to assess the effect of further potential incomplete collections using 24-hour creatinine excretion.

Analyses were replicated after excluding participants with Meas-24HUCrea less than $3 \mathrm{mmol}$ or greater than $25 \mathrm{mmol}$ in women or less than $6 \mathrm{mmol}$ or greater than $30 \mathrm{mmol}$ in men [28]. Statistical analyses were carried out using Statistical Package for the Social Sciences (SPSS, Inc., Chicago, IL, USA) and plots were built in Xlstat 2014.

\section{Results}

Basic characteristics of the study's population are shown in table 1. Overall, mean age and BMI $( \pm \mathrm{SD})$ were 48.9 years $( \pm 15.1)$ and $27.7 \mathrm{~kg} / \mathrm{m}^{2}( \pm 5.6)$, respectively. The mean 24 -hour urine sodium excretion was $2847 \mathrm{mg}$ /day (SD; 1372) equivalent to a salt intake of $7.2 \mathrm{~g}$ /day $(1 \mathrm{~g}$ of $\mathrm{Na}+$ is equivalent to $2.54 \mathrm{~g} \mathrm{NaCl})$ [29]. Except for mean age $(\mathrm{p}=0.042)$ and urinary volume $(\mathrm{p}=0.038)$, there are no significant differences in baseline characteristics and urinary measurements between the training data set and the validation data set (table 1).

Table 1: Basic characteristics of the study population

\begin{tabular}{|c|c|c|c|c|}
\hline & $\begin{array}{c}\text { Overall } \\
(\mathrm{N}=371)\end{array}$ & $\begin{array}{c}\text { Training } \\
\text { data set } \\
(\mathrm{N}=183)\end{array}$ & $\begin{array}{c}\text { Validation } \\
\text { data set } \\
(\mathrm{N}=188)\end{array}$ & $\mathrm{P}$ value \\
\hline Age (years) & $\begin{array}{c}48.9 \\
(15.1)\end{array}$ & $50.4(15.6)$ & $47.4(14.5)$ & 0.042 \\
\hline Height (cm) & $\begin{array}{l}163.7 \\
(8.6)\end{array}$ & $163.8(8.7)$ & $163.6(8.4)$ & 0.954 \\
\hline Weight (kg) & $\begin{array}{c}74.0 \\
(14.4)\end{array}$ & $73.9(14.5)$ & $74(14.3)$ & 0.786 \\
\hline BMI (kg/m²) & $27.7(5.6)$ & $27.7(5.7)$ & $27.7(5.5)$ & 0.800 \\
\hline SBP (mmHg) & $\begin{array}{l}132.4 \\
(20.7)\end{array}$ & $\begin{array}{l}132.2 \\
(21.4)\end{array}$ & $\begin{array}{l}132.7 \\
(20.1)\end{array}$ & 0.631 \\
\hline DBP (mmHg) & $\begin{array}{c}80.2 \\
(11.8)\end{array}$ & $80.0(11.5)$ & 80.5 (12.2) & 0.532 \\
\hline \multicolumn{5}{|l|}{$\begin{array}{l}\text { Urinary } \\
\text { measurements } \\
\text { 24-h urine } \\
\text { collection }\end{array}$} \\
\hline $\begin{array}{l}\text { Sodium } \\
\text { (mg/day) }\end{array}$ & $\begin{array}{l}2847.1 \\
(1372)\end{array}$ & $\begin{array}{c}2808.8 \\
(1561.1)\end{array}$ & $\begin{array}{c}2936 \\
(1319.3)\end{array}$ & 0.108 \\
\hline $\begin{array}{l}\text { Creatinine } \\
\text { (mg/day) }\end{array}$ & $\begin{array}{c}1096.9 \\
(421)\end{array}$ & $\begin{array}{l}1088.6 \\
(451.1)\end{array}$ & $\begin{array}{l}1128.6 \\
(403.2)\end{array}$ & 0.142 \\
\hline $\begin{array}{l}\text { Urinary } \\
\text { volume (L) }\end{array}$ & $1.38(0.6)$ & $1.33(0.57)$ & $1.42(0.62)$ & 0.038 \\
\hline $\begin{array}{l}\text { Spot urine } \\
\text { collection }\end{array}$ & & & & \\
\hline $\begin{array}{l}\text { Sodium } \\
(\mathrm{mg} / \mathrm{L})\end{array}$ & $\begin{array}{c}2796.2 \\
(1495.9)\end{array}$ & $\begin{array}{l}2746.5 \\
(1397)\end{array}$ & $\begin{array}{c}2844.6 \\
(1587.7)\end{array}$ & 0.363 \\
\hline
\end{tabular}




\begin{tabular}{lllll}
$\begin{array}{l}\text { Creatinine } \\
(\mathrm{mgl} / \mathrm{L})\end{array}$ & $\begin{array}{c}1116.6 \\
(582.6)\end{array}$ & $\begin{array}{c}1156.9 \\
(629.1)\end{array}$ & $\begin{array}{c}1077.5 \\
(532.1)\end{array}$ & 0.251 \\
\hline
\end{tabular}

Values are mean standard deviation.

BMI, Body mass index calculated using paired t-test.

all values are calculated using bootstrap.

The model regression coefficients derived from the training data set are shown in Table 2.

Table 2: The newly Developed prediction model

PredU-Na24-h (mg/day)
$=1341-13.8$ Age (years) +
$\begin{aligned} & 27.5 \text { BMI }(\mathrm{m} 2 / \mathrm{kg})+0.56 \text { Spot urinary Na }(\mathrm{mg} / \mathrm{L})- \\ & 0.129 \quad \text { Spot urinary } \mathrm{Cr}(\mathrm{mg} / \mathrm{L})\end{aligned}$

Abbreviations: BMI, body mass index $(\mathrm{m} 2 / \mathrm{kg}) \mathrm{R}^{2}=0.274$; Adjusted $\mathrm{R}^{2}=0.258$

Concentrations of spot urine sodium and creatinine, age, and, BMI were included based on the documented influence of these variables on the sodium urine excretion [30-13-16] and their significant contribution in the model. Coefficients were positive for BMI, and spot urine sodium concentration, but negative for age and, spot urine creatinine concentration (table 2). The adjusted $\mathrm{R}^{2}$ was 0.258 . The newly developed model from the training data set was used to predict 24-hour urine sodium excretion in the validation data set.

Overall, the mean bias calculated using the paired ttest, as the predicted 24-h sodium excretion minus measured 24-h sodium excretion was small and not significant (-17.8 mg/day; $\mathrm{SD}=1357 ; p=0.751)$ (table 3).

Table 3: Mean bias and correlations between measured and predicted 24-hour urinary sodium excretion

\begin{tabular}{|c|c|c|c|}
\hline & $\begin{array}{c}\text { Overall } \\
(\mathrm{N}=188)\end{array}$ & $\begin{array}{c}\text { Men } \\
(\mathrm{N}=69)\end{array}$ & $\begin{array}{r}\text { Women } \\
(\mathrm{N}=119)\end{array}$ \\
\hline Mes-NaU-24h & 2936.0 & 3017.2 & 2888.7 \\
\hline$(\mathrm{mg} / \text { day })^{\mathrm{a}}$ & (1319.3) & (1488.3) & (1214) \\
\hline Pre-NaU-24h & 2918.1 & 2824.1 & 2972.6 \\
\hline$(\mathrm{mg} / \text { day })^{a}$ & $(905.4)$ & $(801.2)$ & (959.8) \\
\hline Mean difference & -17.8 & -193.0 & 83.8 \\
\hline (mg/day) & $(1357.2)$ & $(1407.8)$ & $(1322.4)$ \\
\hline $\begin{array}{l}\text { P-value of } \\
\text { difference }^{b}\end{array}$ & 0.751 & 0.259 & 0.491 \\
\hline Pearson & 0.431 & 0.508 & 0.414 \\
\hline correlation & {$[0.258-$} & {$[0.362-$} & {$[0.160-$} \\
\hline coefficient $^{\mathrm{c}}$ & $0.580]$ & $0.638]$ & $0.614]$ \\
\hline $\begin{array}{l}\text { Pearson } \\
\text { correlation P- } \\
\text { value }\end{array}$ & $<0.001$ & $<0.001$ & $<0.001$ \\
\hline
\end{tabular}

Abbreviations; Mes-NaU-24h: Measured-24-hour urinary sodium excretion. Pre-NaU-24h: Predicted-24-hour urinary sodium excretion; ${ }^{a}$ Values are mean (Standard deviation); ${ }^{b}$ calculated using paired t-test; ${ }^{\mathrm{c}}$ Values are Pearson correlation coefficient and 95\% Confidence Interval (95\%CI) 95\% CI, calculated using bootstrap.

Considering the gender difference, we found a nonsignificant mean bias of underestimation of the measured 24-h sodium excretion (-193 mg/day; $\mathrm{SD}=1408 ; p=0.259)$ for men. While for women, there was a mean bias of overestimation of $84 \mathrm{mg} /$ day $(\mathrm{SD}=$ 1322 but also non-significant $(p=0.491)$. The Pearson correlation coefficient between the pred $24 \mathrm{hUNa}$ and meas $24 \mathrm{hUNa}$ wass 0.431 [ $95 \% \mathrm{CI}, 0.258-0.580$ ]; 0.508 [95\%CI, 0.362-0.638] for men and 0.414 [95\%CI, $0.160-0.614$ ] for women (all $\mathrm{p}<0.001$ ) (table 3).

Overall, the scatter plot of the measured versus the predicted 24-hour urinary sodium excretion showed an $\mathrm{R}^{2}$ of 0.194 and an adjusted $\mathrm{R}^{2}$ of 0.190 (figure 1 ). The adjusted $\mathrm{R}^{2}$ was 0.247 for men, and 0.165 for women (data not shown). Several data points did not cluster around the identity line, indicating individual errors produced by the newly developed prediction model, however these data points were evenly distributed in both sides of the identity line, which explains the small mean bias in the measurement of the $24 \mathrm{hUNa}$. The negative individual differences are compensated by the positive ones resulting in a small mean bias at the group level (figure 1).

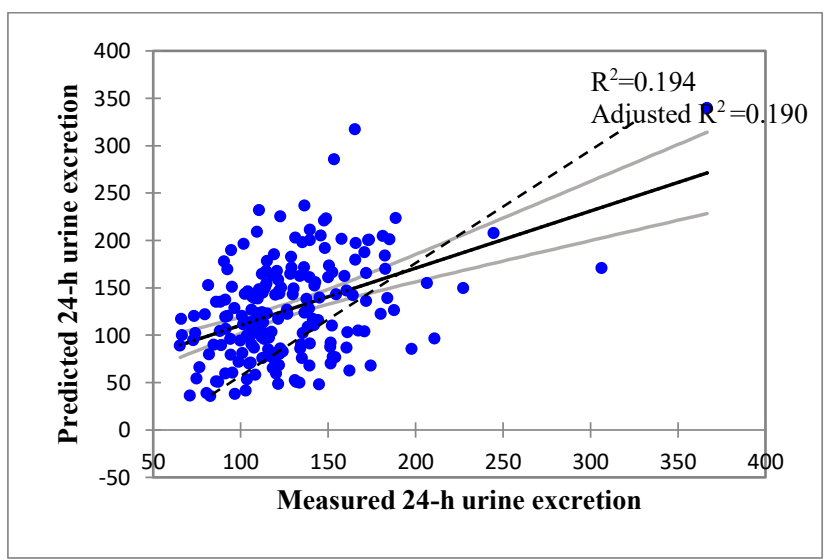

Fig.1: Measured and predicted 24-h urinary sodium excretion in the validation data set.

The regression line with the $95 \%$ confidence interval $(95 \% \mathrm{CI})$ of the measured against predicted 24-h urinary sodium excretion were represented by a solid lines. The line of identity was represented by a dashed line.

The Bland-Altman plot showed a small nonsignificant mean bias (predicted-measured) between the two measurements of the $24 \mathrm{HUNa}(-17.785 \mathrm{mg}$; $95 \% \mathrm{CI},-213$ to 177.5$)$, but the limits of agreement were wide (lower limit $=-2678 \mathrm{mg} /$ day, upper limit $=2642$ $\mathrm{mg}$ /day) (figure 2). Individual differences along the 24$\mathrm{h}$ sodium excretion distribution showed a systematic bias overestimating sodium intake at lower 24 hour sodium excretion and underestimating at higher excretion (figure 2). 
Both genders showed a nonsignificant small mean bias between the two sodium measurements $(-193$ $\mathrm{mg} /$ day; $95 \% \mathrm{CI},-531$ to 145 for men vs $8 \mathrm{mg} /$ day; $95 \% \mathrm{CI},-156$ to $324 \mathrm{mg} /$ day for women), with wide limits of agreement for men (-2952 mg/day; 2566 $\mathrm{mg}$ /day) and women (-2508 mg/day; $2676 \mathrm{mg} /$ day $)$ (data not shown). The same pattern of an overestimation trend at lower 24-h sodium excretion and underestimation at higher levels was observed in men and women (data not shown).

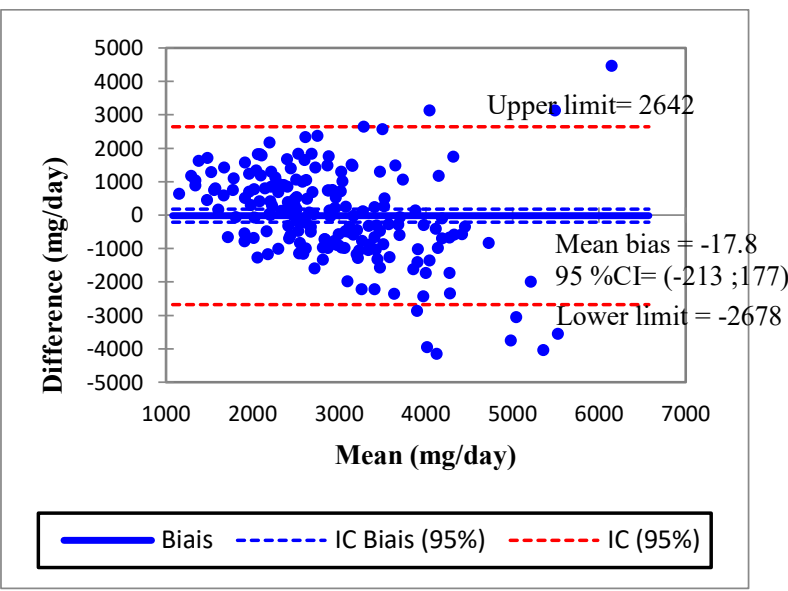

Fig. 2: Bland Altman plot to assess bias produced by the newly developed model to predict 24 -h urinary sodium excretion presented for the total sample.

Bias was calculated as the absolute differences (predicted minus measured) in 24-h urinary sodium excretion. The $95 \%$ limit of agreement are bias $\pm 1.96 \times \mathrm{SD}$.

Completeness of 24-hour urine collections in the present study was assessed using a standard protocol based on rigorous examination of the collection period, total urine volume and a post-collection questionnaire completed by participants. This approach has been used in the International Cooperative Study on Salt, Other Factors, and Blood Pressure (INTERSALT) [31], and recently in the National Health and Nutrition Examination Survey (NHANES) [32]. In addition, we excluded further participants with creatinine excretion values outside the range of $6 \mathrm{mmol} / \mathrm{day}, 30 \mathrm{mmol} /$ day for men, and $3 \mathrm{mmol} / \mathrm{day}, 25 \mathrm{mmol} / \mathrm{day}$ for women [28], but the results remain unchanged.

\section{Discussion}

This study was designed to develop a Moroccan-specific model to estimate $24 \mathrm{HUNa}$ using a single spot urine specimen. The newly developed model underestimates the mean $24 \mathrm{HUNa}$ at the group level by a small nonsignificant bias of $-17.8 \mathrm{mg} /$ day. At the individual level, differences in the predicted and measured $24 \mathrm{HUNa}$ was inconsistent through the level of $24 \mathrm{HUNa}$, with a systematic tendency of overestimation at lower level of 24HUNa and underestimation at higher level.
Research examining the utility of spot urine as a simpler and easier to implement method to replace 24hour urine collection, which is accurately the reference method for estimating sodium intake in individual and population, are of great importance as studies suggested that the 24-hour urine collection may be a barrier to estimate sodium intake, particularly in poor countries [33].

The inclusion of spot urine sodium and creatinine concentrations, age, and BMI was based on knowledge of the influence of these variables on sodium excretion $[34,35,36,37]$, and on their significant contribution to the model. We used a one-step (direct) regression to derive an estimate for $24 \mathrm{HUNa}$, similar to the INTERSALT equation developed based on data from North American and European participants of the INTERSALT study [38].

The direct regression approach used in this study is considered more appropriate compared to the two-steps regression approach used by Tanaka, Kawasaki and Mage, which consist of estimating the sodium-tocreatinine ratio in the 24-hour urine from sodium-tocreatinine ratio in the spot urine and estimate of 24HUCrea from sex, weight, height, age, and in some cases, ethnicity, since the removal of one step should reduce statistical error in the estimation procedure [38].

Our findings show that the developed model produced a small and non-significant mean bias of underestimation, overall. The mean difference indicates an underestimation bias for men and an overestimation bias for women. The reason for the change of the bias direction between genders remain unclear and could be explained by differences in sodium intake, the way the kidney handles sodium excretion differently in men and women, or differences in the parameters used to develop this model.

We found a coefficient of correlation between the measured and predicted $24 \mathrm{HUNa}$ of 0.431 [95\%CI, $0.258-0.580]$. This was lower correlation than those reported in previous studies [39], [38]. By comparison, the Tanaka and Kawasaki equations were developed and validated in Japanese populations based on a relatively higher correlations of $0.540-0.730$, but no data on agreement were reported. Assessment of the agreement in means, and the variability of differences across individuals is the ideal approach to examine the validity of a new method to be used interchangeably or to replace another method [40]. The use of correlation to assess agreement of two methods has been widely criticized [41] [42].

Our following was to examine bias using the BlandAltman method, which is the reference method for 
assessing method agreement [43] [44]. Similar to the INTERSALT equation, we found a small nonsignificant mean bias (-17.8 $\mathrm{mg} /$ day (SD;1357) $(p=0.751))$ which suggest the validity of the newly developed model based on a single spot urine to estimate mean sodium intake of the Moroccan population. The wide limits of agreement (lower limit $=-2678 \mathrm{mg} / \mathrm{day}$, upper limit $=2642 \mathrm{mg}$ /day) and the systematic bias of overestimating sodium intake at lower $24 \mathrm{HUNa}$ excretion and underestimating intake at higher excretion shown in the current study confirm the findings of previous studies suggested that predictive model are inadequate to estimate $24 \mathrm{HUNa}$ at the individual level [34] [38] [45] [46] [47]. This was not unexpected, the sodium intake of an individual varies highly from meal to meal, and the sodium concentration in spot urine specimens has been shown to reflect the sodium content in food and beverages consumed within hours of the urine collection [48]. This diurnal intraindividual variability in sodium excretion is a source of systematic error when estimating $24 \mathrm{HUNa}$ using spot urine [33][48].

This is, to our knowledge, the first study to estimate 24HUNa in Moroccan population using a model based on intrinsic coefficients. However, our study had some limitations. Firstly, completeness of 24-hour samples is the major problem of the method. We used the WHO protocol to assess sodium concentration in 24-hour urine collection [49]. Standard detailed instructions were provided and a post-collection interview was conducted with each participant to exclude eventual under or over-collected urine samples based on a doubtful period of collection, loss of urine, and urine volume. Moreover, we conducted a sensitivity analysis using a criteria for $24 \mathrm{HUCrea}(<3 \mathrm{mmol} /$ day or $>25$ $\mathrm{mmol} /$ day in women and $<6 \mathrm{mmol} /$ day or $>30$ $\mathrm{mmol} /$ day in men [52]), and the results remain unchanged. However, it is always possible that there are still potential incomplete collections in the final sample. Ensuring the completeness of collections is crucial for an accurate estimate of sodium intake. The use of reference Biomarkers such as paraaminobenzoic acid is recommended, however, it is impractical and burdensome to be used in population studies[17], particularly in low-middle income countries. Measured creatinine or ratio of measured-topredicted creatinine rate has been used to assess completion of 24-hour urine, but there are currently no standard criteria. Although relatively constant, creatinine excretion is influenced by the differences between populations in protein intake pattern, in muscle mass, and ethnicity [17]. To our knowledge, for now there is no specific equation to predict $24 \mathrm{HUCrea}$ in the Moroccan population. Therefore, the use of predictive equations of creatinine developed in different sitting could wrongly distinguish collections according to their completion in our population. Secondly, in the current study, only a single spot specimen collected at the evening was used.

Sodium excretion vary across the day, and studies have shown inconsistency in the validity of the most frequently used predictive equations of $24 \mathrm{HUNa}$ depending on the time of spot urine [50] [51]. Investigators suggested collecting spot urine at random times of the day to compensate for the potential systematic error that could be produced due to the circadian rhythm of the $24 \mathrm{HUNa}$ [33]. We admit that the ideal would have been to collect spot urine at different time of the day in order to choose the best model. However, the choice of evening spot urine in the present study was based on metabolic studies showed that sodium excretion is maximal during the afternoon and evening and minimal from midnight to early in the morning [52] [53] [54].

In addition to numerous validation studies [39] [55] have shown that the highest prediction accuracy was with equations based on the afternoon and evening spot as compared to the morning or overnight spot.

Findings from the current study have an important implication for future studies to estimate sodium intake in Moroccan population and monitor the trend of its change over time, which is a key information to assess the effectiveness of strategies implemented to reduce salt intake in populations. Our study support the use of a specific newly developed model based on a single spot urine concentrations and participants' basic characteristics to approximate the mean 24-h urinary sodium excretion for population, but not for individuals.

To summarize, by using a single spot urine which is more suitable and easier to collect than 24-hour urine, the newly developed model was found reliable to approximate the mean population sodium intake. But the performance at the individual level was poor.

\section{Conflict of Interest}

All authors declare no conflict of interest.

\section{Funding}

The author(s) received no financial support for the research, authorship and/or publication of this article.

\section{Acknowledgements}

The authors are grateful to all the participants and would like to thank all investigators and staff of Joint Research Unit in Nutrition and Food-Rabat for helping with the laboratory analysis and the data collection. 


\section{References}

1. F. P. Cappuccio, Cardiovascular and other effects of salt consumption. Kidney Int Suppl., 3:312-5 (2013).

2. F. He, J. Li and G. Macgregor. Effect of longerterm modest salt reduction on blood pressure: Cochrane systematic review and meta-analysis of randomised trials. BMJ., 346, f1325 (2013).

3. A. Mente, M. O'Donnell, S. Rangarajan et al., Association of urinary sodium and potassium excretion with blood pressure. N Engl J Med 371, 601-11. (2014)

4. Burden Global of Disease Website; http://vizhub.healthdata.org/gbd-compare/ (2018). Accessed in May 3, 2018.

5. I. Brown, I. Tzoulaki, V. Candeias, et al. Salt intakes around the world: implications for public health. Int J Epidemiol; 38: 791-813 (2009).

6. D. Mozaffarian, S., Fahimi, G. M. Singh, R. Micha, S. Khatibzadeh, R. E. Engell, ... and J. Powles. Global sodium consumption and death from cardiovascular causes. New England Journal of Medicine, 371(7), 624-634 (2014).

7. Guideline: Sodium Intake for Adults and Childrene. World Health Organization; Geneva, Switzerland: (2012).

8. WHO. Noncommunicable diseases and mental health. About 9 voluntary global targets (2020). https://www.who.int/nmh/ncd-tools/definitiontargets/en/

9. Ministère de la Santé. Stratégie Nationale Multisectorielle de Prévention et de Contrôle des Maladies Non Transmissibles 2019 - 2029 http://www.emro.who.int/fr/mor/morocconews/lancement-de-la-strategie-nationale multisectorielle-de-prevention-et-de-controle-des-maladiesnon-transmissibles.html (2019). Accessed 28 Mai 2021

10. M. O’Donnell, A. Mente, S. Yusuf. Sodium intake and cardiovascular health. Circ Res.; 116:104657 (2015).

11. Centers for Disease Control and Prevention. Sodium reduction toolkit: a global opportunity to reduce population-level sodium intake. (2013).

12. WHO. Global action plan for the prevention and control of noncommunicable diseases 2013-2020. Geneva (Switzerland) (2013).

13. I.J. Brown, A.R. Dyer, Q. Chan et al. Estimating 24-hour urinary sodium excretion from casual urinary sodium concentrations in Western populations: the INTERSALT study. Am J Epidemiol 177, 1180-92 (2013).

14. T. Kawasaki, K. Itoh, K. Uenzo, et al. A simple method for estimating $24 h$ urinary sodium and potassium excreation from second morning voiding urine specimen. Clin Exp Pharmacol Physiol; 20: 7-14 (1993).
15. U. Toft, C. Cerqueira, A. H. Andreasen, B. H. Thuesen, P. Laurberg, L. Ovesen, ... and T. Jørgensen, Estimating salt intake in a Caucasian population: can spot urine substitute 24-hour urine samples?. European journal of preventive cardiology, 21(10), 1300-1307 (2014).

16. T. Tanaka, T. Okamura, K. Miura et al. A simple method to estimate populational 24-h urinary sodium and potassium excretion using a casual urine specimen. J Hum Hypertens. 16(2):97-103 (2002).

17. J. Polonia, M. F. Lobo, L. Martins et al Estimation of populational 24-h urinary sodium and potassium excretion from spot urine samples: evaluation of four formulas in a large national representative population. J Hypertens 35, 477-486 (2017).

18. M. E. Cogswell, C. Y. Wang, T. C. Chen et al Validity of predictive equations for 24-h urinary sodium excretion in adults aged 18-39 y. Am J Clin Nutr. 98, 1502-13 (2013).

19. A. Mente, M. J. O'Donnell, G. Dagenais et al Validation and comparison of three formulae to estimate sodium and potassium excretion from a single morning fasting urine compared to 24-h measures in 11 countries. J Hypertens. 32, 1005-14; discussion 1015 (2014).

20. K. Charlton, L. J. Ware, G. Chidumwa, M. Cockeran, A. E. Schutte, N. Naidoo and P. Kowal, Prediction of 24-hour sodium excretion from spot urine samples in South African adults: a comparison of four equations. Journal of human hypertension, 1 (2019).

21. R. McLean, S. Williams and J. Mann. Monitoring population sodium intake using spot urine samples: validation in a New Zealand population. J Hum Hypertens. 28, 657-62 (2014).

22. Ministre de la santé du Royaume du Maroc, 2019. Rapport de l'Enquête Nationale sur les Facteurs de Risque communs des Maladies Non Transmissibles, STEPS, (2017 - 2018).

23. WHO/PAHO. Regional Expert Group for Cardiovasular Disease Prevention through Populationwide Dietary Salt Reduction, Protocol for Population Level Sodium Determination in 24-hour Urine Samples; World Health Organization: Geneva, Switzerland, (2010).

24. The WHO STEPwise approach to noncommunicable disease risk factor surveillance. Geneva: World Health Organization (2017). http://www.who.int/chp/steps/STEPS_Manual.pdf?ua= 1

25. World Health Organization. STEP wise approach to surveillance (STEPS) (2012); http://www.who.int/chp/steps/en/ Accessed October 1 st, 2020 
26. J. M. Bland, D. G Altman. Statistical methods for assessing agreement between two methods of clinical measurement. Lancet; 1:307-10 (1986).

27. J. M. Bland, D. G Altman. Applying the right statistics: analyses of measurement studies. Ultrasound Obstet Gynecol; 22:85-93 (2003).

28. M. A. Wenxia et al., Validation and Assessment of Three Methods to Estimate 24-h Urinary Sodium Excretion from SpotUrine Samples in High-Risk Elder Patients of Stroke from the Rural Areas of Shaanxi Province. Int. J. Environ. Res. Public Health, 14, 1211 (2017); doi:10.3390/ijerph14101211

29. Y. Peng, W. Li, Y. Wang, H. Chen, J. Bo, X. Wang, L. Liu, Validation and Assessment of Three Methods to Estimate 24-h Urinary Sodium Excretion from Spot Urine Samples in Chinese Adults. PLoS ONE (2016).

30. J. V. Joossens, J. Claessens, J. Geboers et al. Electrolytes and creatinine in multiple 24-hour urine collections (1970-1974). In: Kesteloot H, Joossens JV, eds. Epidemiology of Arterial Blood Pressure. The Hague, Netherlands: Martinus Nijhoff Publishers; 4563 (1980).

31. P. Elliott, R. Stamler. Manual of operations for INTERSALT, International cooperative study on the relation of sodium and potassium to blood pressure. Control. Clin. Trials. 9:1617S (1988)

32. M. E. Cogswell, C. M. Loria, , A. L. Terry, L. Zhao, C. Y. Wang, T. C. Chen, ... and L. J. Appel. Estimated 24-hour urinary sodium and potassium excretion in US adults. Jama, 319(12), 1209-1220 (2018).

33. M. E. Cogswell, J. Maalouf, P. Elliott et al., Use of Urine Biomarkers to Assess Sodium Intake: Challenges and Opportunities. Annu Rev Nutr 35, 34987 (2015).

34. W. M., Vollmer, F. M., Sacks, J. Ard, L. J. Appel, G. A. Bray, D. G. Simons-Morton et al., Effects of diet and sodium intake on blood pressure: subgroup analysis of the DASH-sodium trial. Ann Intern Med; 135: 10196028 (2001). https://doi.org/10.7326/00034819-135-12-200112180-00005

35. Intersalt Cooperative Research Group. Intersalt: an international study of electrolyte excretion and blood pressure. Results for 24 hour urinary sodium and potassium excretion. BMJ; 297:319e328 (1988).

36. A. C. Baxmann, M. S. Ahmed, N. C. Marques et al. Influence of muscle mass and physical activity on serum and urinary creatinine and serum cystatin $C$. Clin J Amer Soc Nephrol. 3(2):348_354 (2008).

37. L. Zhou, J. Stamler, Q. Chan, L. Van Horn, M. L. Daviglus, A. R. Dyer, ... and P. Elliott. Salt intake and prevalence of overweight/obesity in Japan, China, the United Kingdom, and the United States: the INTERMAP Study. The American Journal of Clinical Nutrition,
110(1), 34-40 (2019)

38. I. J. Brown, A. R. Dyer, Q. Chan et al. Estimating 24-hour urinary sodium excretion from casual urinary sodium concentrations in Western populations: the INTERSALT study. Am J Epidemiol 177, 1180-92 (2013).

39. S. J. Mann and L. M. Gerber. Estimation of 24hour sodium excretion from spot urine samples. J Clin Hypertens 12, 174-80 (2010).

40. K. J. Van Stralen, K. J. Jager, C. Zoccali and F. W. Dekker. Agreement between methods. Kidney international, 74(9), 1116-1120 (2008).

41. J. M. Bland, D. G. Altman. Measuring agreement in method comparison studies. Stat Meth Med Res. 8:135660 (1999).

42. U. Toft, L. Kristoffersen, S. Ladelund et al. Relative validity of a food frequency questionnaire

43. R. M., McLean, V. L. Farmer, A. Nettleton et al. Twenty-Four-Hour Diet recall and Diet records compared with 24-hour urinary excretion to predict an individual's sodium consumption: A Systematic Review. J Clin Hypertens 20, 1360-1376 (2018).

44. J. M. Bland, D. G. Altman. Statistical methods for assessing agreement between two methods of clinical measurement. Lancet 1, 8476, 307-10 (1986).

45. E. Emeville, C. Lassale, K. Castetbon, V. Deschamps, B. Salanave, M. Vernay, ... and E. KesseGuyot. Estimating sodium intake from spot urine samples at population level: a validation and application study in French adults. British Journal of Nutrition, 122(2), 186-194 (2019).

46. R. M. McLean. Measuring population sodium intake: a review of methods. Nutrients 6, 4651-62 (2014).

47. L. Zhou, Y. Tian, J. J. Fu et al. Validation of spot urine in predicting 24-h sodium excretion at the individual level. Am J Clin Nutr 105, 1291-1296. (2017).

48. N. R. Campbell, F. J. He, M. Tan, F. P. Cappuccio, B. Neal, M. Woodward, ... and P. Whelton. The International Consortium for Quality Research on Dietary Sodium/Salt (TRUE) position statement on the use of 24_hour, spot, and short duration (< 24 hours) timed urine collections to assess dietary sodium intake. The Journal of Clinical Hypertension, 21(6), 700-709 (2019).

49. R. Wilks and J. Kingston. Protocol for population level sodium determination in 24-hour urine samples (2010).

50. M. Wenxia et al, Validation and Assessment of Three Methods to Estimate 24-h Urinary Sodium Excretion from Spot Urine Samples in High-Risk Elder Patients of Stroke from the Rural Areas of Shaanxi Province. Int. J. Environ. Res. Public Health, 14, 1211 (2017); doi:10.3390/ijerph14101211

51. M. E. Cogswell, C. Y. Wang, T. C. Chen, C. 
M. Pfeiffer, P. Elliott, C. D. Gillespie, A. L. Carriquiry, C. T. Sempos, K. Liu, C. G. Perrine, C. A. Swanson, K. L. Caldwell, C. M. Loria. Validity of predictive equations for 24-h urinary sodium excretion in adults aged 18-39 y. Am J Clin Nutr; 98:15026513 (2013).

52. IOM (Institute of Medicine). Sodium Intake in Populations: Assessment of Evidence. Washington, DC: The National Academies Press; (2013).

53. B. Bentley. A review of methods to measure dietary sodium intake. J Cardiovasc Nurs; 21:63-7 (2006).

54. A. J. Clark, S. Mossholder. Sodium and potassium intake measurements: dietary methodology problems. Am J Clin Nutr; 43:470д76 (1986).

55. N. B. Allen, L. Zhao, C. M. Loria, L. Van Horn, C. Y. Wang, C. M. Pfeiffer et al. The Validity of predictive equations to estimate 24-h sodium excretion: the MESA and CARDIA urinary sodium study. Am J Epidemiol; 186:149 P159 (2017). 